\title{
An Intellectual Brain MRI Image Retrieval System to aid the Diagnosis of Brain Tumors
}

\author{
Chethan K, Rekha Bhandarkar
}

\begin{abstract}
Human brain being a complex organ, detecting abnormalities like Brain Tumor, Alzheimer, and Schizophrenia etc. are not an easy task. A computer aided automated system called Content Based Medical Image Retrieval System (CBMIR) can be used to assist the medical practitioner in arriving at correct diagnosis. Brain tumor is a kind of disease related to the brain malfunction and goes through various stages. Brain tumor identification, classification in its initial stages is an important and challenging task. In this paper, focus is on revival of brain tumor images from large database where multiple stages of diseases are present. In order to realize this task, a feature extraction technique comprising of Local Binary Pattern (LBP), Gabor, and Histogram of Gradient (HOG) are used. Based on the attributes, Support Vector Machine (SVM) classifier is used for pattern learning and classification. An experiment is performed to measure the accuracy of SVM classifier and performance of the CBMIR system for different classes of brain tumor disease. With the extracted attributes, SVM achieves an accuracy of $89.33 \%$, average precision of $89.35 \%$ and recall of $89.33 \%$.
\end{abstract}

Keywords--- CBMIR; Brain Tumor, SVM classifier, Feature Extraction, Euclidean Distance, Manhattan distance.

\section{INTRODUCTION}

In the digitized world with the advancement of the technology complex problems can be encountered which need to be solved in a simple way. A picture can never be portrayed by negligible words, consequently there is a requirement for an approach where images without the content labels or names are retrieved. Brain tumor is a major contributor for the death rate increase in the world. Tumor can be either benign or malignant. Benign tumors are non-harmful wherein the seriousness of the infection advances gradually without influence the encompassing tissues, whereas malignant tumors are cancerous-cells which are quickly developing and influence the rest of the parts of the brain. If the tumor is identified in the earlier stages the patient can be saved and by studying the stages of the tumors from previous patients, a solution can be found for diagnosing. Hence, meticulous classification and distinction between benign and malignant tumor is required for treatment and follow-ups. MRI images are frequently taken to identify the existence of the tumor. For each patient, sequences of images are taken from particular views and at different orientation. The data so produced is stored in Picture Archiving and Communication System (PACS). The data access and surfing, searching for the stored cases in
PACS is a tedious job because the retrieval is done using the information on the patient which may be his/her id, age, gender or name. In such a system the images are glossed with the characteristic of the tumor or the diagnostic report. The gloss requires a human to analyze and provide the label based on his intelligence and experience. The technique so utilized is inclined to complications, tedious, erroneous as the human view varies starting with one then onto the next. A Content Based Retrieval (CBIR) approach where the images serve as query can overcome the above stated problem. The images of the patients are collected and the features associated with images such as texture, shape can be found which allows the representation of images in terms of its features. The extracted features are stored along with the image which serves as a means for measuring the closeness of the images to the query images.

\section{LITERATURE SURVEY}

Several researches have been conducted in the field of CBIR for various applications. Few papers which are closely relevant to the proposed work are presented as follows:

GayatriChavanet. Al. [1] developed a technique for revival of brain MRI images wherein the features were extracted by GLCM and the highlighted features were delivered for selection using Sequential Forward Selection (SFS) technique. The combination of SVM-KNN classifiers were used wherein the classifier distinguishes the MRI images into normal, noncancerous and malignant and Euclidian measure was utilized to retrieve the images from the relevant classified class.

Amit Kumar Rohithet. al. [2] has presented an advanced method for CBIR where alongside the revival procedure, tumor was also detected. To accomplish this, 2D wavelet features were extracted and characterization for the normal and tumor cases was done by SVM. The tumor was identified using Incremental Supervised Neural Network (ISSN) and invariant moments.

Abderrahimkhatabi et.al. [3] have discussed a shape based approach for CBIR wherein the Angular Radial Transform (ART) has been used for feature extraction. The segmentation is accomplished by applying K- Nearest Neighborhood (KNN) method followed by extracting visual features of shape using the ART coefficients. The obtained features were subjected to SVM classifier to decide if the tumor is available or not. Since the difference in the values of ART coefficients serves as a measure of closeness, the 


\section{AN INTELLECTUAL BRAIN MRI IMAGE RETRIEVAL SYSTEM TO AID THE DIAGNOSIS OF BRAIN TUMORS}

existence of tumor is recognized by KNN strategy.

Akila and Uma maheswari [4] have developed a CBIR system applying binary association rules which involved a pre-processing stage for noise removal and features were extracted using moment invariant features which resulted in feature vectors. In the testing stage the query image feature vectors are classified by using Artificial Neural Network (ANN) as normal, benign, malignant. Similarity measure is checked using trigonometric function distance.

$\mathrm{K}$ Yuan et. al. [5] have proposed a framework for CBIR system which involved the database collection from the hospitals and storing it to the database for retrieval purpose, a GUI is created which in turn is connected to a search engine through web server. The search engine searches the database for the similar images by comparing Non-Negative Tensor Factorization (NTF) features with the query image NTF features. The accuracy and efficiency of the feature extraction method using NTF is found to be better than Gabor, Principle Component Analysis (PCA) and Non-negative Matrix Factorization (NMF). The major disadvantage of the discussed methods are making the physicians use either manual marking of the abnormal region or for deciding and refining of the feature set and also use of text along with images for revival.

From this discussion, it is noticed that, though considerable work has been accomplished to improve the medical diagnostic systems however the key issues associated to image indexing, complexity of feature extraction, performance of retrieval are challenging tasks which need to be addressed. Henceforth, in this work it has been attempted to address the above stated drawbacks and an approach is presented for brain Tumor MRI image retrieval. The main contributions of this work is to extract the attributes of images, creating attribute directory and storing attribute vector in attribute directory followed by training the SVM classifier to correctly identify the class and retrieving the data similar to query.

\section{METHODOLOGY}

The flow diagram of the CBIR system for the determination of brain tumor is depicted in Fig. 1.

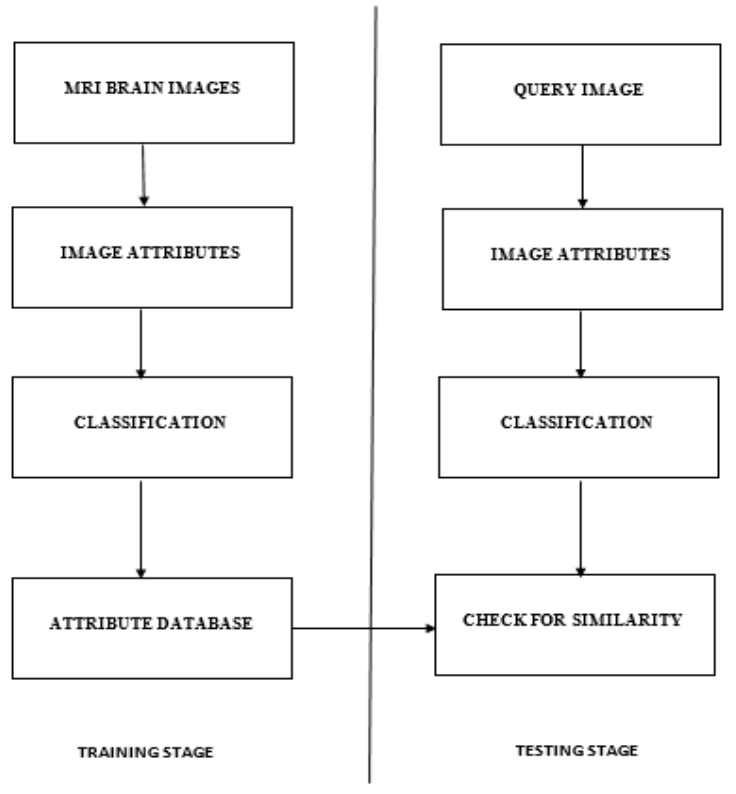

Fig. 1: Methodology
It involves 2 phases: offline (training) and online (testing) phase. In offline phase, the tumor images saved in the database are segmented by eliminating skull region by performing morphological operations to find the tumor region of interest followed by extracting Gabor, HOG, and LBP features representing the different classes of the segmented tumor. The feature set corresponds to each class are fed to SVM classifier to get the class label as benign and malignant. The attributes of each class label are saved in an attribute directory. Equivalently, the online phase deals with applying query image arbitrarily and segmenting it followed by extracting the features. After this, the distance between attribute vectors of query image is compared with [12] that of attributes in the directory using Euclidean and Manhattans distance metrics to retrieve the identical images of brain tumor.

\section{SEGMENTATION}

Segmentation is an initial step performed before any of the processing application. The tumor region is segmented using fuzzy type-2 [6]. The skull region of the image is removed by performing morphological operations. To achieve this task, firstly a curved shaped structuring element is chosen with which the images are eroded and then the output of erosion is subjected to expansion. In each iteration, disk size is augmented to perform erosion and expansion. The iteration is stopped when only the skull region is obtained. The difference is taken between the obtained skull from the original image to get the skull removed image. Since the tumor images contain grey matter, white matter, Cerebrospinal Fluid (CSF), tumor region. These 4 parts are considered as a membership function to determine the probability of pixel associated to a specific group [6]. The trapezoidal approximation is used for determination of the membership functions [18] as shown in Fig 2.

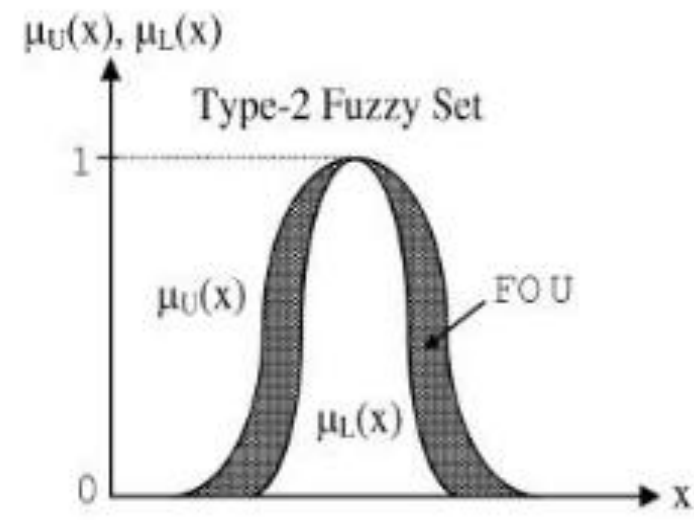

Fig. 2: Distribution of Fuzzy type-2 set.

If $(a i j, b i, c i j, d i j)$ is in $\mathrm{i}^{\text {th }}$ membership function for $\mathrm{j}^{\text {th }}$ input Then, output membership precedent is given by

$O i=(a i, b i, c i, d i)$. The paradigm used for $\mathrm{j}^{\text {th }}$ is as in eqn 1. 


$$
A_{i j}\left(x_{j}\right)=\left\{\begin{array}{cc}
\frac{x_{j}-a_{i j}}{b_{i j}-a_{i j}} & \text { if } a_{i j}<x_{j}<b_{i j} \\
1 & x \geq 0 \\
\frac{d_{i j}-x_{j}}{d_{i j}-c_{i j}} & \text { if } c_{i j}<x_{j}<d_{i j}
\end{array}\right.
$$

The values of the edges of the trapezium must satisfy $a i j<b i j<c i j<d i j$ and fused whenever the distance between the values are very less. The trapezoidal consisting of the membership function is moved along with the histogram of the image and the values are computed and compared with that of the membership values. The output of the segmentation consisting of four matrices of the pixel values of the corresponding membership functions.

\section{FEATURE EXTRACTION}

Feature extraction is appropriate in the situation whenever the system is unable to process the enormous number of redundant input data. Hence it is required to represent the features by performing transformation to consider only the relevant set of features thereby minimizing the effort required to handle the input for processing. The high level information like color, texture and shape could be extracted from the images and this high level information is significant for the classifier during the training and testing stage. This work aims at extracting texture attributes of tumor images.

\section{1)Gabor Features}

The Gabor attributes gives the texture attribute of the brain tumor image. These attributes are extracted using the 2D Gabor bank of filters for diverse scale and orientation [7]. The filter bank can be defined as in eqn 2 [7].

$$
H(x, y)=\frac{1}{2 \pi \sigma_{x} \sigma_{y}} e^{\left[-\frac{1}{2}\left(\frac{x^{2}}{2}+\frac{y^{2}}{\sigma_{x}^{2}} \sigma_{y}^{2}\right)+2 \pi j \mathrm{~F}\right]}
$$

Where $\mathrm{F}=$ modulation frequency, $\sigma_{x}^{2}=$ orientation along $\mathrm{x}$ axis, $\sigma_{y}^{2}=$ Orientation along y axis.

Function alike in eqn (2) results from dilating and revolving the mother function

1

$$
H(x, y)=b^{-2 k} h\left(x^{\prime}, y^{1}\right), b \geq
$$

$\mathrm{X}^{\wedge \prime}$

$=\mathrm{b}^{\wedge}(-\mathrm{k})(\& \mathrm{x} \cos \theta$

$+\mathrm{y} \sin \theta)$

$$
\text { 『 } \quad \mathrm{y}^{{ }^{\prime}}=\mathrm{b}^{\wedge}(-\mathrm{k})(-\mathrm{x} \sin \theta+\mathrm{y} \sin \theta)
$$

(5)

where $\mathrm{k}$-scale, $\mathrm{k}=0 \ldots . \mathrm{K}-1, \quad \theta=\frac{\mathrm{l} \pi}{\mathrm{L}}, \mathrm{b}=$ scale factor, $\mathrm{L}=$ direction

The filter outputs are obtained by performing convolution between the results and image

H_kl

$=\mathrm{A}(\mathrm{x}, \mathrm{y})$

$* \mathrm{~h} \_\mathrm{kl}(\mathrm{x}, \mathrm{y})$

$=\frac{1}{\mathrm{UXV}} \sum_{\mathrm{x}} \sum_{\mathrm{y}}\left|\mathrm{H}_{\mathrm{kl}}{ }^{\mu_{\mathrm{kl}}}(\mathrm{x}, \mathrm{y})\right|$

$$
=\sqrt{\frac{1}{\mathrm{UXV}}} \sum_{\mathrm{X}} \sum_{\mathrm{Y}}\left(\left|\mathrm{H}_{\mathrm{kl}}(\mathrm{x}, \mathrm{y})\right|\right.
$$

Where $\mu_{k l}=$ mean, $\mathrm{U} \times \mathrm{V}=$ image size, $\sigma_{k l}=$ standard deviation

The attribute vector is obtained as,

1))]

$$
\mathrm{GF}=\left[\mu_{-} 00, \sigma_{-} 00, \mu_{-} 01, \ldots \ldots . \mu_{-}((k-1)), \sigma_{-}((L-\right.
$$

\section{2)Local Binary Bit pattern}

LBP provides a binary pattern for each pixel and algorithm is presented as follows [12] :

S1: Divide an image into smaller blocks

S2: For each pixel in the block, a bit pattern is found by performing the below mentioned steps:

1. Bit pattern is found by considering 8-neighborhood pixels either in clockwise and anticlockwise directions

2. If center pixel is less than neighborhood, assign zero ('0') else assign one (' 1 ')

3. A binary string is collected known as bit pattern

4. Count the number of each bit pattern

5 . Keep only uniform bit pattern and normalize it

S3: Arrange uniform bit pattern and count the number of occurrences occurring in the block in a column

S4: Appending all columns of all blocks to form Bit Pattern (BP) matrix which is the feature of the image

The LBP can be summarized in the eqn 10

$$
=\sum_{\substack{\left.\mathrm{u}=0 \\-\mathrm{p}_{\mathrm{c}}\right)}}^{\mathrm{U}-1} \mathrm{~T}\left(\mathrm{p}_{\mathrm{u}}\right.
$$$$
\text { (B. P) })_{\mathrm{UV}}
$$

Where $U=$ neighbors associated with the center pixel located in the specified radius $\mathrm{V} . \mathrm{p}_{\mathrm{u}}=$ grey value of the neighbor pixel. $\mathrm{p}_{\mathrm{c}}=$ grey value of the pixel considered as center.

\section{3)Histogram of Gradients}

HOG are also called as Edge Histogram descriptors (EHD). In this method, an image is partitioned into 8832 smaller blocks of $4 \times 4$ and corner orientation is calculated in 5 directions. Then feature vector is formed by collecting five features of each smaller image block. The HOG is calculated in 4 directions $0,45,90,135$ degrees [7]. The way of dividing an image into blocks and sub blocks is shown in Fig. 3

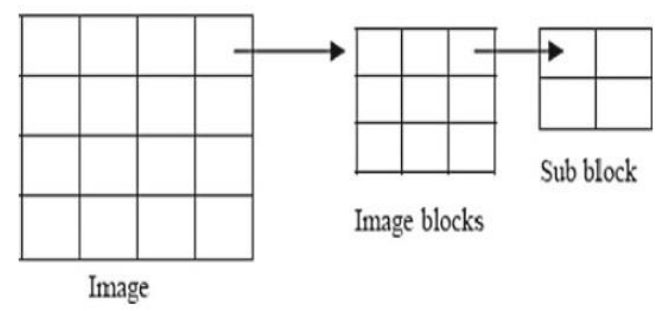

Fig. 3: Image partition

The distribution of corners along 4 different directions [10] is as shown in Fig. 4. 


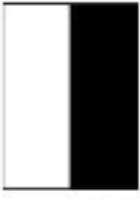

(a)

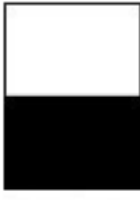

(b)

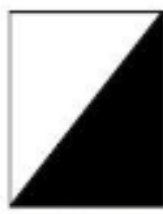

(c)

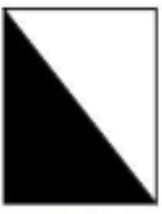

(d)

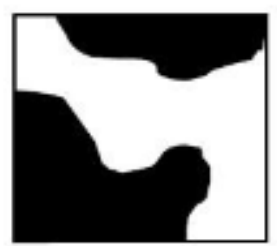

(e)
Fig. 4: Distribution of Edges (a) Vertical (b) Horizontal (c) $45^{\circ}$ (d) $135^{\circ}$ (e) non-directional

The filters used for the calculation of EHD are as shown in Fig.5

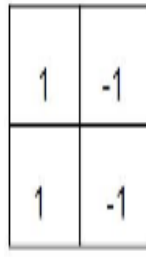

(a)

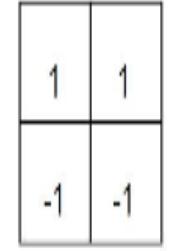

(b)

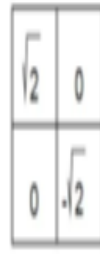

(c) (d)
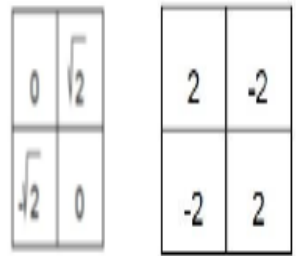

(e)
Fig. 5: Filters for (a) vertical (b) Horizontal (c) $45^{\circ}$ (d) $135^{\circ}(\mathrm{e})$ non directional

The filters are used to plot the HOG for each sub-block to yield scruples of bins and a total of 80 bins are used for image which is partitioned into $4 \times 4$ sub-blocks. The obtained bins are shown in Fig. 6

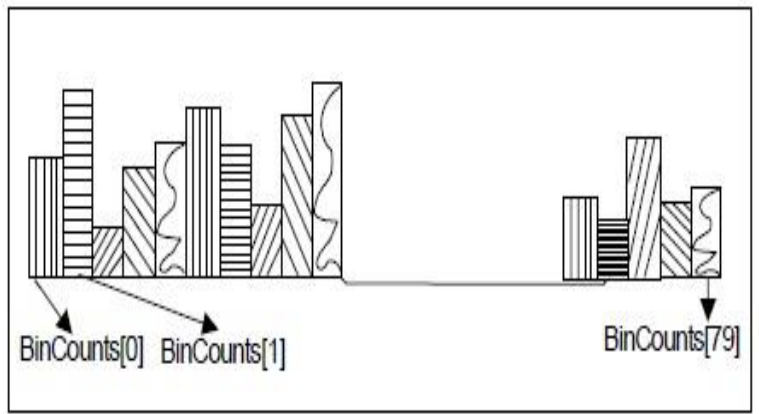

Fig. 6: Bins along 5 directions

\section{CLASSIFICATION}

The classifier used is SVM classifier. The main idea behind this classifier is that it tries to find finest hyperplane to differentiate the attributes. SVM's are primarily a 2 class classifier wherein the boundaries between the linear and nonlinear classes can be learned. Since the SVM involves training stage, delivering known data with decision values to an SVM is significant to form a training set. The SVM uses Langrange's theorem to find the best hyperplane as shown in Fig 7.

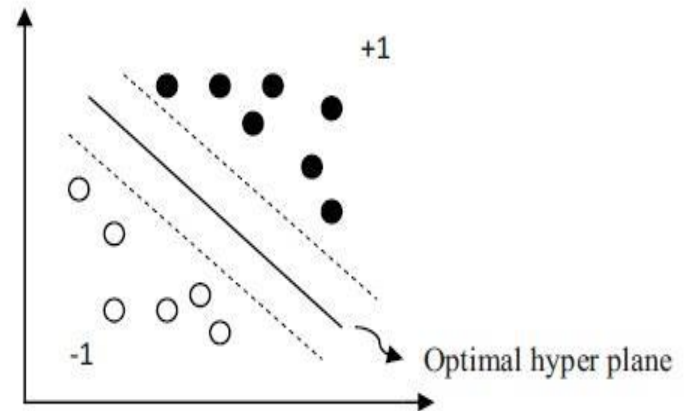

Fig.7: Hyperplane

The optimality is defined by,

$$
c_{j} \cdot\left(v^{T} a_{j}+s\right) \text { for } \mathbf{j}
$$

$=1,2, \ldots . M$

Where $\mathbf{a}_{\mathbf{j}}=$ training sample, $\mathbf{M}=$ total number of training samples, vT $=$ weight vectors, $\mathrm{s}$ is bias, $c j=+1$ or -1 .

The weight vectors possess value such that it minimizes

$\frac{1}{2} v^{T} \mathbf{v}$

$$
\boldsymbol{\theta}(\mathbf{v})=
$$

where $\boldsymbol{\theta}(\mathbf{v})=$ cost Function, v=weights

The overall function for best solution is as

$\mathbf{F}(\mathbf{v}, \mathbf{s}, \boldsymbol{\alpha})$

$=\frac{1}{2} \mathbf{v}^{\mathrm{T}} \mathbf{v}$

$-\sum_{j=1}^{M} \alpha_{j}\left[\left(v^{T} a_{j}+s\right)\right.$

-1]

Where $\boldsymbol{\alpha}_{\mathbf{j}}=$ Langrang's multipliers

The Radial Basis Function (RBF) kernel functions are used to simplify the calculation. There may be cases where the classifications maybe nonlinear in such cases kernel function are used for calculation. The function for non-linear function is given by

$\mathbf{f}(\mathbf{a})$

$=\operatorname{sign}\left(\sum_{j=1}^{M} \alpha_{j} b_{j} k\left(a, a_{j}\right)\right.$

$+\mathbf{s})$

Where $\mathbf{a}_{\mathbf{j}}=$ input, $\mathbf{b}_{\mathbf{j}}=+1$ or $-1, \mathrm{~s}=$ bias, $\mathrm{k}=$ kernel, $\mathbf{k}(\mathbf{a}, \mathbf{b})=$ $\mathbf{e}^{\left[\frac{\|\mathbf{a}-\mathbf{b}\|^{2}}{2 \boldsymbol{\sigma}^{2}}\right]}, \boldsymbol{\sigma}=$ constant.

\section{SIMILARITY MEASURE}

Similarity measurement is a crucial step in a CBMIR system where identical images are retrieved based on image inquired by the user. The performance of revival system relies upon the measurement of closeness between the attributes of an image in the directory and query image attributes. Therefore, Euclidean and Manhattans distance computation techniques are utilized for closeness measurement. Euclidean distance is the most effective technique wherein the distance between the attribute of query image and attribute vectors in the attribute directory are computed by calculating the summation of squared

Published By:

Blue Eyes Intelligence Engineering

\& Sciences Publication

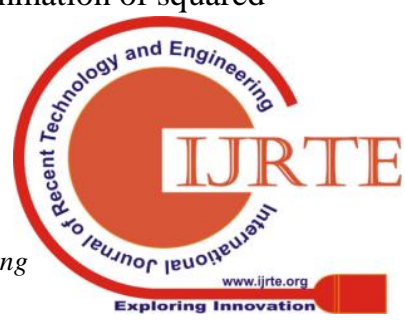


differences and then taking square root to retrieve the identical images. It can be computed as in eqn 15 .

$$
\boldsymbol{d}=\sqrt{\sum_{\mathrm{I}=\mathbf{1}}^{\mathrm{N}}\left(\mathbf{E}_{\mathbf{j}}-\mathbf{I}_{\mathbf{j}}\right)^{2}}(15)
$$

The Manhattans distance is popularly known as city block distance where the distance is computed by taking summation of differences with the query image attribute and attributes vectors in the directory. This can be calculated as in eqn 16.

$$
\mathbf{D}=\sum_{\mathbf{i}=\mathbf{1}}^{\mathrm{N}}\left|\mathbf{E}_{\mathbf{j}}-\mathbf{I}_{\mathbf{j}}\right|
$$

Where $E_{j}=$ query attribute vector, $I_{j}=$ database attribute vector

\section{RESULTS AND DISCUSSION}

The developed retrieval scheme is implemented on the dataset containing brain tumor images of 300 patient data wherein each patient data consists of 50 images acquired in different views and series. Since these images were in dicom format, it has been converted into jpg using microdicom viewer tool. A database is created by selecting 150 benign and 150 malignant T2 images of different patient. Few sample images of dataset are depicted in Fig. 8.

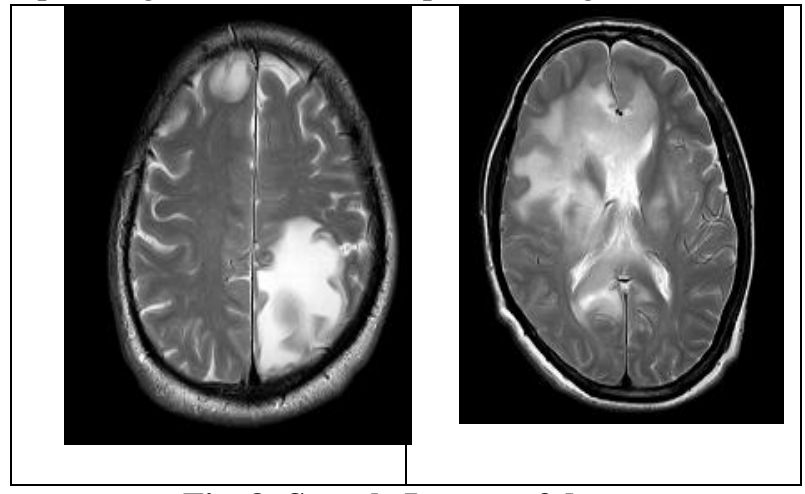

Fig. 8: Sample Images of dataset

In this work, Firstly, all the images in the database are segmented for tumor region identification and then attributes of Gabor, LBP, and HOG descriptors are extracted and vectors are stored in attribute directory as .mat file. Next, a random query image is provided and subjected to segmentation process followed by creating attribute vector. Ten images were retrieved and queried. The attributes of query and attributes in the directory are fed to SVM classifier. Finally, the 10 images similar to query are returned as depicted in Fig. 9.

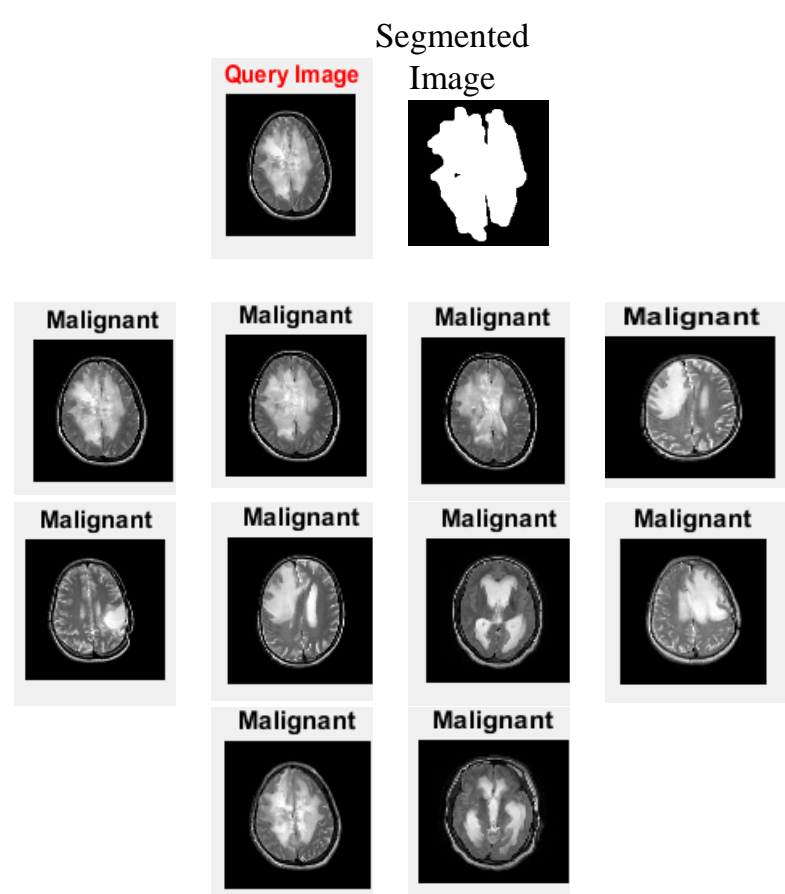

Fig. 9: Returned Images

The performance of the classifier is quantified by the following metrics [8].

$$
\begin{aligned}
& \text { Accuracy }=\frac{\mathrm{TP}}{\mathrm{TP}+\mathrm{TN}+\mathrm{FN}+\mathrm{FP}} \\
& \text { Specificity }=\frac{\mathrm{TN}}{\mathrm{TN}+\mathrm{FP}} \\
& \text { Sensitivity }=\frac{\mathrm{TP}}{\mathrm{TP}+\mathrm{FN}}
\end{aligned}
$$

Where $\mathrm{TP}=$ True positive, $\mathrm{TN}=$ True Negative, $\mathrm{FN}=$ False Negative, $\mathrm{FP}=$ False Negative.

The effectiveness of the revival system is measured using precision and rememberance (Recall) rate. This can be computed as:

$$
\frac{\text { Number of relevant images retrived }}{\text { Number of images retrived }} \text { Precision }=
$$

Recall =

Number of relevant images in the database

Where TP and TN indicate how many images are properly classified based on the classes. FP denotes misclassified results by giving noncancerous images as cancerous. TN gives the noncancerous images as noncancerous. FN indicates total number of cancerous images which are misclassified as noncancerous. These terminologies are characterized as a matrix known as confusion matrix. The results of SVM-RBF kernel in terms of confusion matrix is depicted in Table 1. 
Table 1: Confusion Matrix-SVM-RBF kernel

\begin{tabular}{|l|l|l|}
\hline Class & Benign & Malignant \\
\hline Benign & 68 & 7 \\
\hline Malignant & 9 & 66 \\
\hline
\end{tabular}

Several statistical measures can be quantified by the confusion matrix inorder to assess the SVM performance and the same has been presented in Table 2 .

Table 2: Performance of SVM and CBMIR system

\begin{tabular}{|c|c|c|c|c|c|}
\hline Class & $\begin{array}{l}\text { Sensitiv } \\
\text { ity } \\
(\%)\end{array}$ & $\begin{array}{l}\text { Specific } \\
\text { ity }(\%)\end{array}$ & $\begin{array}{l}\text { Precisi } \\
\text { on } \\
(\%)\end{array}$ & $\begin{array}{l}\text { Reca } \\
11 \\
(\%) \\
\end{array}$ & $\begin{array}{l}\text { Accur } \\
\text { acy } \\
(\%)\end{array}$ \\
\hline Benign & 90.66 & 86.66 & 88.31 & 90.66 & \multirow{2}{*}{$\begin{array}{l}89.33 \\
\%\end{array}$} \\
\hline $\begin{array}{l}\text { Maligna } \\
\text { nt }\end{array}$ & 88 & 90.66 & 90.41 & 88 & \\
\hline
\end{tabular}

From table 2, the feature extraction techniques are assessed with the SVM with accuracy of $89.33 \%$ with improved sensitivity and specificity. These two parameters are compared for both the classes of the tumors as depicted in Fig.10.

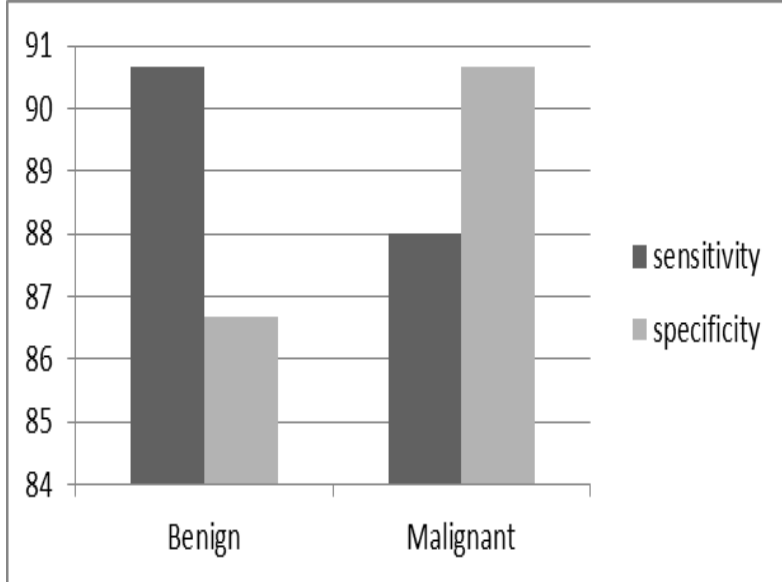

Fig. 10: Comparative analysis of sensitivity and specificity

From the comparative study of sensitivity and specificity, it shows that this classifier predicts the benign and malignant cases almost equally which is especially very important for diagnosis.

The performance comparision of CBMIR are analyzed using precision and recall rate and presented graphically as shown in Fig. 11.

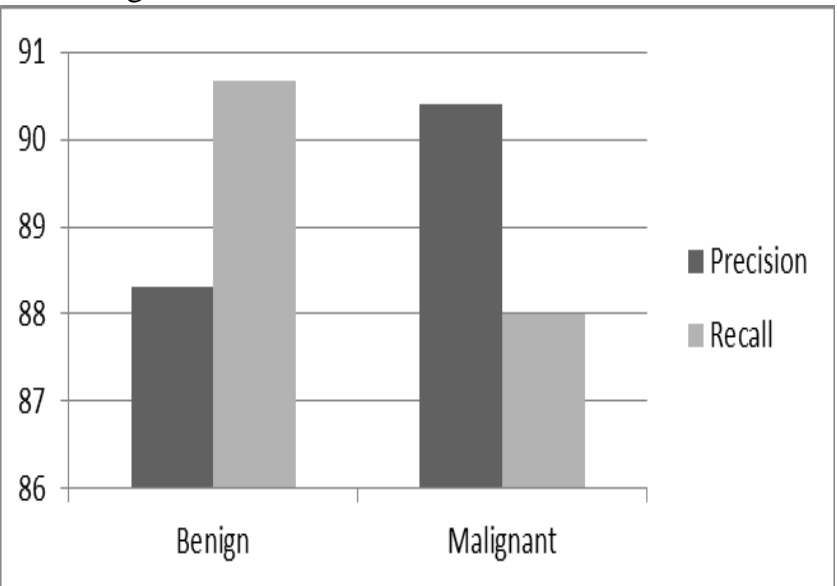

Fig. 11: Performance of CBMIR system
From this study, the attributes extracted using LBP, Gabor, HOG achieves the precision of $88.31 \%, 90.4 \%$ for benign class and recall rate of $90.66 \%$ and $88 \%$ for malignant class respectively.

\section{CONCLUSION}

In this paper, a method for retrieval of brain tumor images has been carried based on the classification and similarity matching. The aim was to correctly classify the query to one of the classes, namely benign and malignant, and retrieve the corresponding images in the classified set. The proposed method correctly identifies the class of the image, but while retrieving it is seen that the other class images are also retrieved due to misclassification and distance measurement technique. It is observed that the SVM classifier yields a satisfactory average sensitivity, specificity of $89.33 \%$ and $88.49 \%$ for both benign and malignant classes with an overall accuracy of $89.33 \%$ and also the developed retrieval scheme outperforms with an average precision of $89.35 \%$ and average recall of $89.33 \%$ for benign and malign classes. The accuracy of the classification and performance of the CBIR system can be enhanced by increasing the number of samples in the training stage

\section{REFERENCES}

1. GayatriChavan, Sonal gore, " Two tier Approach for Automatic retrieval of MRI Brain image by Feature Extraction,"International Journal of Computer Applications, Vol. 132-No 14, pp: 46-48, 2015

2. Amit Kumar Rohit, N.G. Chitaliya, "A Novel Approach for Content based Mri Brain Image Retrieval," International Journal of Soft Computing and Engineering, Vol.4, Issue-3, pp.2231-2307, 2014

3. AbderrahimKhatabi, AmalTmiri, Ahmed Seshir, "An effective System for Content MRI Brain Image Retrieval using Angular radial Transform,"'International Journal of Computer Applications, Vol. 117-No.24,pp: 29-32, 2015

4. Akila, Uma Maheswari, "Content Based medical Image retrieval using Binary Association rules,"Journal of Computer Science, Vol.9, no. 6, pp:678-689, 2013

5. K. Yuan, Z. Tian, J. Zou, Y. Bai, and Q. You, "Brain CT image database building for computer-aided diagnosis using content-based image retrieval," Inf. Process. Manag., vol. 47, no. 2,pp. 176-185, 2010

6. R. Nicole, DeepaliAneja, Tarun Kumar rawat, “ Fuzzy Clustering Algorithms for Effective medical Image Segmentation," International Journal of intelligent systems and applications, Vol. 11, pp:55-61, 2013

7. MeghaP.Arakeri, G.RamMohanareddy, "An intelligent content-based image retrieval system for clinical decision support in brain tumor diagnosis," International Journal of Multimedia Information Retrieval, Vol.2, pp:175-188, 2013

8. Sudheesh K V, L Basavaraj, " Impact of Statistical Texture Feature Abstraction based Classification applied for Detection of Abnormalities in Brain CT Images," International Journal of pure and Applied Mathematics, Vol. 118, No.18, pp: 2645-2654, 2018

9. Hui Zhang, Quing Ming JonathanWu,YuhuiZheng, Thanh Minh Nguyen, "Effective Fuzzy Clustering algorithm with Bayesian model and mean template for image segmentation," IET Image Processing ,Vol. 8, 
Issue10, pp 571-581, 2014

10. Chee Sun Won, Dong Kwon Park, Soo-Jun Park, "Efficient use of MPEG-7 Edge Histogram Descriptor,"ETRI Journal, Vol.24, No 1, pp: 23-30, 2002

11. A Kannan, "Content Based Image Retrieval Using Nearest Neighbour and Hybrid KNNSVM Methods to Diagnose MR Images," International Journal of Innovative Research in Science, Engineering and Technology, Vol. 3, No 3, pp. 3021-3026, 2014

12. Megha P. Arakeri, G.Ram Mohan Reddy," Medical Image Retrieval System For diagnosis of brain tumor bassed on classificaion and content similarity," 2012 Annual IEEE India Conference (INDICON),pp:416-421, 2012

13. Jai-Andaloussi, Lamard M, CazuguelG, .'Content Based Medical Image retrieval Based OnBEMD: Optimisation Of Similaritymetric," $32^{\text {nd }}$ Annual International conference of the IEEE Engineering in Medicine and Biology, pp: 3069-3072, 2010

14. M. S. Sudhakar and K. BhoopathyBagan, "An effective biomedical image retrieval framework in a fuzzy feature space employing Phase Congruency and GeoSOM," Appl. Soft Comput. J., vol. 22, pp. 492-503, 2014

15. Li Song, Xiabi Liu, Ling Ma1, Chunwu Zhou, Xinming Zhao, YanfengZhao."Using HOG-LBP Features and MMP Learning to Recognize Imaging Signs of lung lesions," $25^{\text {th }}$ IEEE International Symposium on Computer-Based Medical System (CBMS),2012

16. B.Ramamurthy, K.RChandran, V.R. Meenakshi, V.Shilpa."Content based medical image retrieval system using texture and intensity for dental images,"Eco-friendky Computing and Communication Systems, ICECCS, Vol. 305, pp:125-134, 2012

17. Abraham Varghese, KannanBalakrishnan, Reji R. Varghese, joseph S. Paul."content based image retrieval of T2 weighted brain MR images similar to T1 weighted images," Pattern Recognition and Machine Intelligence, Springer Nature America, Inc, pp: 474-481, 2013

18. M.EminYuksel Senior Member,IEEE and Murat Borlu."Accurate Segmentation Of Dermoscopic images by thresholding based on type 2 Fuzzy logic," IEEE Transactions on Fuzzy Systems, Vol.17, no.4, pp:976-982, 2009 